\title{
A three-dimensional model of spiral null pair to form ion-scale flux ropes in magnetic reconnection region observed by Cluster
}

Cite as: Phys. Plasmas 26, 112901 (2019); https://doi.org/10.1063/1.5114620

Submitted: 10 June 2019 . Accepted: 11 October 2019 . Published Online: 01 November 2019

Ruilong Guo (D), Zuyin Pu, Zhonghua Yao, Malcolm Dunlop, Yulia V. Bogdanova, Yong Wei, Xiaogang Wang (D), Chijie Xiao, Jiansen He, Suiyan Fu (D), Lun Xie, Andrew N. Fazakerley (D), and Weixing Wan

\section{ARTICLES YOU MAY BE INTERESTED IN}

Gyrofluid modeling of magnetosphere in feedback instability

Physics of Plasmas 26, 112902 (2019); https://doi.org/10.1063/1.5122722

Nernst thermomagnetic waves in magnetized high energy density plasmas

Physics of Plasmas 26, 112702 (2019); https://doi.org/10.1063/1.5122178

Oscillatory regimes of compressible 2.5D viscous linear magnetic reconnection

Physics of Plasmas 26, 112110 (2019); https://doi.org/10.1063/1.5124127

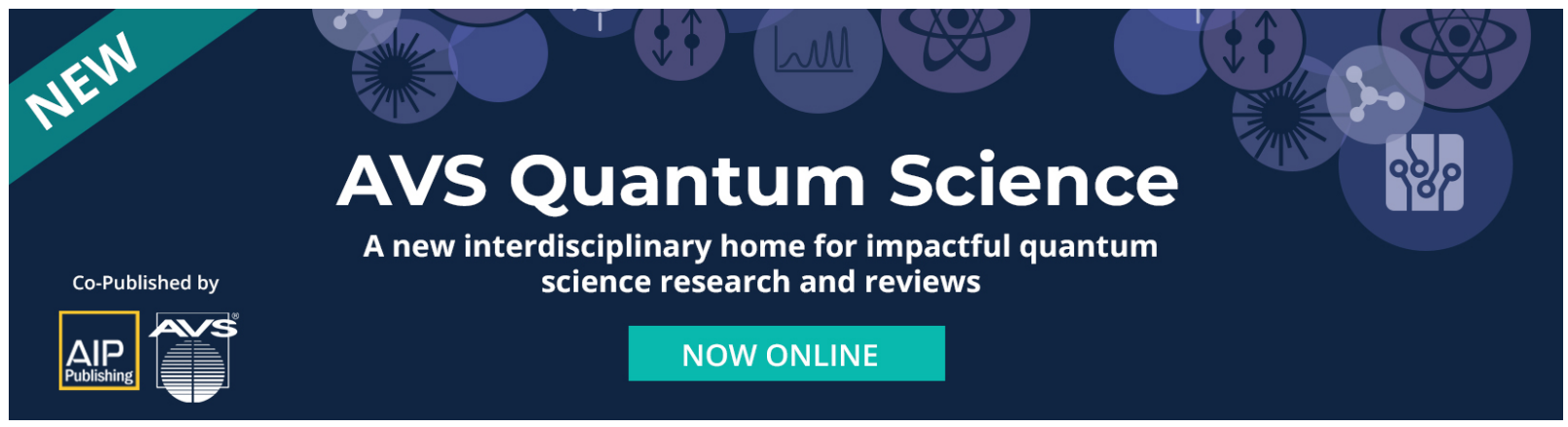




\title{
A three-dimensional model of spiral null pair to form ion-scale flux ropes in magnetic reconnection region observed by Cluster
}

Cite as: Phys. Plasmas 26, 112901 (2019); doi: 10.1063/1.5114620

Submitted: 10 June 2019 - Accepted: 11 October 2019 •

Published Online: 1 November 2019

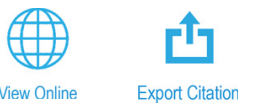

Ruilong Guo, ${ }^{7}$ (D) Zuyin Pu, ${ }^{2}$ Zhonghua Yao, ${ }^{3}$ Malcolm Dunlop, ${ }^{4,5}$ Yulia V. Bogdanova, ${ }^{5}$ Yong Wei, Xiaogang Wang, ${ }^{6}$ iD Chijie Xiao, ${ }^{7}$ Jiansen He, $^{2}$ Suiyan Fu, ${ }^{2}$ (iD Lun Xie, ${ }^{2}$ Andrew N. Fazakerley, and Weixing Wan

\author{
AFFILIATIONS \\ ${ }^{7}$ Key Laboratory of Earth and Planetary Physics, Institute of Geology and Geophysics, Chinese Academy of Sciences, \\ Beijing 100029, China \\ ${ }^{2}$ School of Earth and Space Sciences, Peking University, Beijing 100871, China \\ ${ }^{3}$ Laboratoire de Physique Atmospherique et Planetaire, STAR institute, Universite de Liege, Liege 4000, Belgium \\ ${ }^{4}$ School of Space and Environment, Beihang University, Beijing 100191, China \\ ${ }^{5}$ RAL Space, Rutherford Appleton Laboratory, STFC, Didcot OX11 OQX, United Kingdom \\ ${ }^{6}$ Harbin Institute of Technology, Harbin 150001, China \\ ${ }^{7}$ School of Physics, Peking University, Beijing 100871, China \\ ${ }^{8}$ UCL Mullard Space Science Laboratory, Dorking RH5 6NT, United Kingdom
}

\begin{abstract}
The magnetic structure and topology of the three-dimensional magnetic reconnection region are significantly dynamic and complex. Smallscale flux ropes and magnetic null points are frequently detected in the reconnection outflow region and diffusion region due to the increased in situ measurements at high temporal cadences. Previous studies have demonstrated that X-line and small-scale flux ropes are both related to null points. In this study, by applying a fitting-reconstruction method with the input of the Cluster dataset, we reveal three types of spiral null pairs that serve as the skeleton of the flux ropes. Two spiral nulls can be connected by a spine, or by a separator, or by both a spine and a separator. A theoretical model is proposed to explain these spiral null pairs. The observational results and the model indicate that the number of magnetic loops of the flux rope is restricted by the linkage pattern of two nulls, while the flux rope is confined by the two nulls and their fan surfaces. The model predicts that the magnetic perturbations in the reconnection region can transform the linkage types of the nulls and eventually lead to the evolution of flux ropes.
\end{abstract}

Published under license by AIP Publishing. https://doi.org/10.1063/1.5114620

\section{INTRODUCTION}

Magnetic reconnection (MR) is a fundamental process in plasma environments, which changes the magnetic field configuration and converts magnetic field energy into plasma thermal and kinetic energies. $^{1,2}$ It is widely accepted that in a three-dimensional (3D) MR region, magnetic null points (where the magnetic field vanishes) are pivotal in the process of magnetic field lines' breaking and rejoining ${ }^{3}$ and play a crucial role in the activity regions of the solar corona ${ }^{4}$ and the planetary magnetospheres. ${ }^{5,6}$ The field lines in the vicinity of a $3 \mathrm{D}$ null point constitute a "spine line" where field lines are concentrated and a "fan surface" on which field lines are tiled (clear illustrations for magnetic null points can be found in Figs. 1 and 2 in Ref. 7). Based on the orientation of the field lines on the fan surface and spine line, four types of null points are identified, namely, $A$-null, $A_{s}$-null, $B$-null, and $B_{s}$-null. The $A$-null and $A_{s}$-null are termed as "negative" nulls whose field lines on the fan surface converge to the null point. The $B$-null and $B_{s}$-null are "positive" nulls whose field lines on the fan surface diverge away from the null point. The subscript "s" refers to the "spiral nulls" whose field lines on the fan are spiraled, while the nulls with straight field lines on the fan are termed as radial nulls. The magnetic null points are likely to be formed in pairs ${ }^{8}$ where one corresponds to the negative null, while the other corresponds to the positive null. A wellknown null pair model is termed as the 3D separator MR model. ${ }^{5,7,9-14}$ The fan surfaces of two radial null points intersect at the separator to 
connect the two null points. In the plane perpendicular to the separator, the two-dimensional (2D) $X$-type feature is observed (see the illustration in Fig. 1 of Ref. 5). There have been observations inside Earth's magnetosphere ${ }^{5,11,12,14}$ and at the magnetopause, ${ }^{13}$ which agree with the separator model. The length of the separator can affect the strength of the guide field: a long separator with a sizable magnetic field strength leads to component reconnection, while a short separator with a negligible field strength corresponds to antiparallel MR. ${ }^{14}$ Simulation results also indicate that the separator can stretch across the magnetopause and display component MR features at low latitudes and the antiparallel MR features at high latitudes. ${ }^{15}$

Flux ropes, consisting of twisted magnetic field lines (as shown in Fig. 1 in Ref. 16), are another crucial element in the 3D MR. When the guide field in a flux rope vanishes, the magnetic structure exhibits a closed-loop configuration, which is termed as the magnetic island. Generally, the formation of a magnetic island can result from spatial periodicity in a system like tokamaks, ${ }^{17-19}$ but this is rare in a space environment, which is usually lacking in periodicity. The flux rope structure with a finite guide field is more common in space. The evolution and interaction of flux ropes play important roles in the dynamics and heating of the solar atmosphere. ${ }^{20,21}$ Flux ropes are also observed with a small guide field in Earth's magnetotail between two $X$-lines ${ }^{22}$ and in thin current sheets in the reconnection region. ${ }^{23}$ On the magnetopause, the MHD scale flux rope, i.e., the flux transfer events (FTEs), can transfer mass from the solar wind into the magnetosphere. ${ }^{24}$ Ion-scale flux ropes are also observed on the magnetopause with small current structures. ${ }^{25,26}$ In 3D MR, turbulence and electron physics dominate in the reconnection region, where the flux ropes are generated and interact with each other. ${ }^{27}$ Additionally, the secondary reconnection sites can also form in the reconnection-generated flux ropes and reconnection fronts. ${ }^{28}$

Recent studies indicate that the flux ropes are formed in close relation to spiral null points. ${ }^{29-34}$ In the solar active regions, the spiral nulls can manifest the skeleton of flux ropes where a solar flare occurs. ${ }^{29}$ Flux ropes in association with the spiral null pairs have been detected in the magnetotail. ${ }^{33}$ The spiral nulls can be chained by the spine lines along which flux ropes are formed, while torsional spine reconnection occurs on the spiral nulls. ${ }^{32,33}$ Flux ropes and multiple $3 \mathrm{D}$ null points have also been found in the turbulent reconnection region. ${ }^{34}$ Statistical analysis has been performed on the magnetic nulls observed by the Cluster multiple spacecraft ${ }^{35}$ in the Earth's magnetopause and magnetotail. ${ }^{36,37}$ Magnetic nulls are also reproduced in particle-in-cell simulations of turbulent magnetosheath plasma. ${ }^{32}$ Both observations and simulations indicate that approximately $80 \%$ of the null points correspond to spiral type nulls $\left(A_{s}\right.$ and $\left.B_{s}\right)$, which indicates that the spiral nulls are crucial in the formation of flux ropes and also play an important role in the evolution of the reconnection region. Various linkage types between two null points can exist due to different perturbations during their formation from a degenerated null point. $^{38}$ The topology varies in different types of null pairs, which affects plasma properties inside the flux ropes. It is suggested that a chain of helically wrapped spine linked null pairs can connect two regions with various characteristics and make the flux rope significantly complicated. ${ }^{33}$ The aforementioned extant studies imply that the spiral nulls and the way to link them are important in examining the properties and evolution of flux ropes.

In the present study, we show three different types of null pairs as visualized by reconstruction of the magnetic field data ${ }^{35}$ when Cluster detected flux ropes in the Earth magnetotail. The reconstruction results demonstrate that two spiral nulls can be connected by a spine, or by a separator, or by both and that the field lines between two spiral nulls are twisted and wrapped up to form flux ropes. Thus, we propose an analytical model to illustrate the magnetic field configuration at and around the three types of spiral null pairs and predict that large perturbations in the reconnection region can lead to a mutual transformation between the three types of null pairs. In Sec. II, the observational features and 3D reconstructed pictures of three types of spiral null pairs are presented. The analytical model is shown in Sec. III. Section IV contains the discussion, and the summary is presented in Sec. V.

\section{IN SITU OBSERVATIONS}

In order to investigate the 3D structure of flux ropes, we apply the fitting-reconstruction approach ${ }^{39}$ to the multiple-point magnetic field data from Cluster measurements. ${ }^{35}$ The fitting-reconstruction method is previously used to reconstruct the magnetic field inside and around the Cluster four spacecraft tetrahedron. ${ }^{11-14,33,34}$ Detailed benchmarks ${ }^{14,33}$ indicated that the magnetic topology and essential features of the magnetic fields can be well reassembled with reliability by the fitting-reconstructing technique, when the reconstruction scale does not exceed thrice the tetrahedron size. The magnetic field data we used in the study are from the FGM instrument ${ }^{40}$ on board Cluster, while ion data are from the CIS instrument ${ }^{41}$ and electron data are from the PEACE instrument ${ }^{42,43}$ and the RAPID instrument. ${ }^{44}$ The electron density is derived from spacecraft potential. ${ }^{45}$ The magnetic null points can be indicated by the Poincaré index $(+1$ denotes positive null, -1 denotes negative null, and 0 denotes no null), ${ }^{8,46}$ and the spiral index ${ }^{33}$ can be used to diagnose whether the null point corresponds to a spiral null. If the null corresponds to a radial one, the spiral index is zero; otherwise, the spiral index is set as $+1 /-1$ to represent the As-/Bs- type null. A brief introduction to the Poincare index and the definition of the spiral index are given in Ref. 33. In this section, we show three types of spiral null pairs to form flux ropes.

\section{A. Two spiral nulls paired by the spine to form a flux rope}

A flux rope formed with two spiral nulls in the magnetotail is analyzed in a previous study ${ }^{33}$ where two nulls are paired by their spines and the magnetic field lines are wrapped up to form the flux rope. It is difficult to use the reconstructed magnetic field geometry to distinguish whether the two nulls are linked by their common spine or by two helically wrapped spines although the topology is nearly identical when the two spines are very close to each other. Thus, we termed both situations as the "helically wrapped spine" model and provided the following analytical model for the configuration:

$$
\left(B_{x}, B_{y}, B_{z}\right)=\left[x y-\frac{1}{2} j z+\varepsilon y, 1-y^{2}, z y+\frac{1}{2} j x\right] .
$$

The origin of the coordinates is set at the center of the null-null line. The $\mathrm{Y}$ axis is parallel to the null-null line, which is very close to the dawn to dusk direction for the previously studied events ${ }^{33}$ in the magnetotail. The $\mathrm{X}$ axis is perpendicular to the $\mathrm{Y}$ axis and approximately points toward the Earth, and the $\mathrm{Z}$ axis completes the right-hand orthogonal coordinate systems. In this model, $j$ denotes the current density along the $\mathrm{Y}$ axis and $\varepsilon$ denotes the disturbing degree of the 
magnetic field. The term $\varepsilon y$ represents the magnetic perturbation parallel to the direction pointing from one null to the other. If $\varepsilon=0$, then the two nulls are located at $y= \pm 1$, the spine lines of two spiral nulls lay in the $Y$-direction, and the field lines around the common spine are twisted and exhibit a flux rope structure. When the perturbation term $\varepsilon y \ll 1$, the two nulls appear as connected by their common spine line. ${ }^{33}$

Two events where flux ropes formed with two spiral nulls paired by helically wrapped spine were examined in Ref. 33 . These two events were observed by the Cluster spacecraft in the magnetotail at [-18.7, $3.5,-2.7] \mathrm{R}_{\mathrm{E}}$ (Earth's radius) in GSM coordinates on September 15th, 2001, at 05:01:21.781 UT and at 05:03:24 UT, respectively. They were both detected in the reconnection outflow region albeit with different plasma features due to the formation of the flux ropes. ${ }^{33}$ Figure 1 shows the reconstruction result of the first event (quoted from Fig. 4 in Ref. 33). The electron density is low in both cases, which is typical for the magnetotail. Additionally, we proposed that one of the flux ropes was newly formed in the outflow region, which can locally perturb the plasma environment.

\section{B. Two spiral nulls connected by a separator to form a flux rope}

In addition to the $X$-line, the separator model can also reproduce the flux rope. Figure 2 shows observations of two such flux rope

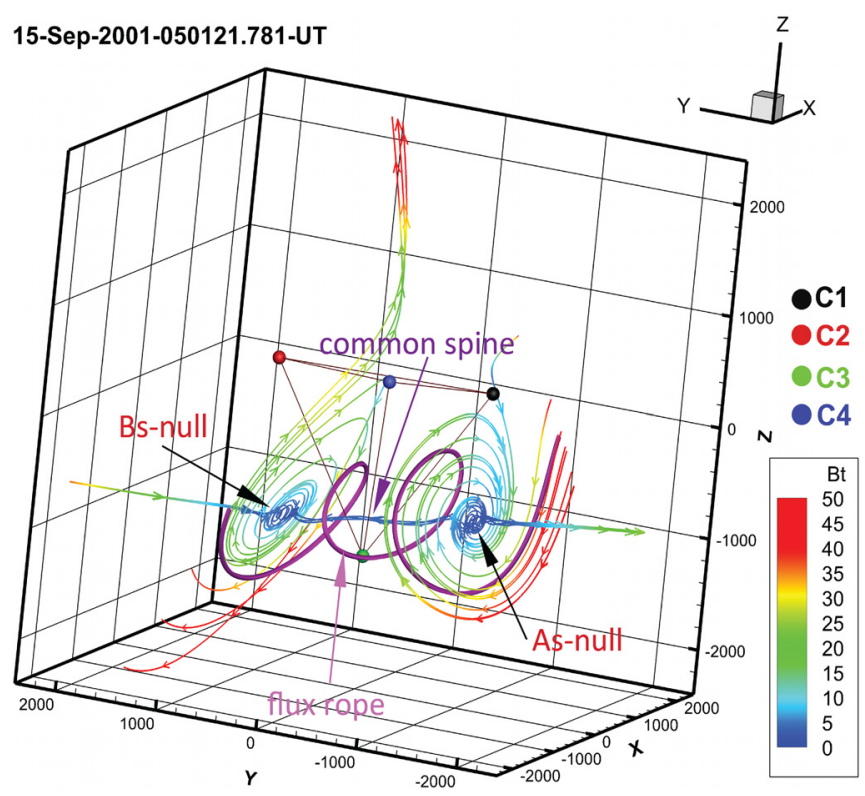

FIG. 1. Reconstruction results for the time at 05:01:21.781 UT. Reproduced with permission from Guo et al., Phys. Plasmas 23(5), 052901 (2016). Copy right 2016 AIP Publishing. ${ }^{33}$ Colored spheres present the location of four Cluster satellites (black, red, green, and blue represent C1, C2, C3, and C4, respectively). The axes of coordinates are parallel to those of the GSM (Geocentric Solar Magnetic) coordinate, while the original point is set at the center of the spacecraft tetrahedron. Colored curves correspond to the constructed magnetic field lines. The arrows on the curves show the direction of the field lines. The configuration consists of a $B_{S^{-}}$ null and an $A_{s}$-null. The two spiral nulls are interlinked by their spine that approximately directs in the Y-direction. The thick purple curve corresponds to the field line to show the flux rope. events. Figures 2(a)-2(c) show the three components of the magnetic field for all four Cluster spacecraft. All spacecraft with the exception of $\mathrm{C} 1$ recorded two bipolar signals (marked by the two vertical dashed lines in Fig. 2) between 09:46:30 UT and 09:48:30 UT on 1st October, 2001 , at $[-16.4,7.9,0.7] \mathrm{R}_{\mathrm{E}}$ in GSM coordinates, which have been studied in previous studies. ${ }^{22,47}$ Furthermore, the electron density [Fig. 2(d)] and the flux of energetic electrons [with energies exceeding $30 \mathrm{keV}$ and below $96 \mathrm{keV}$ as shown in Figs. 2(e)-2(h)] increased. The large $\mathrm{B}_{\mathrm{y}}$ component implied that the magnetic structures correspond to flux ropes but not magnetic islands. When $B_{z}$ changed its sign, the $\mathrm{B}_{\mathrm{x}}$ components detected at all spacecraft remained large and positive, thereby suggesting that Cluster crossed the northern part of the flux rope and did not come into the center region and that the size of the flux ropes should exceed the separation of four spacecraft.

We used the fitting-reconstruction method to diagnose the structure of the flux ropes. Figure 3(a) shows the reconstructed results at 09:47:12 UT (denoted as the first vertical dashed line in Fig. 2). One $A_{s}$-null and one $B_{s}$-null can be found at the southern side of the spacecraft tetrahedron. The fan surfaces of the two spiral nulls intersect to form a separator. Similarly, the reconstruction result at 09:47:47 UT [Fig. 3(b)] during the second $\mathrm{B}_{\mathrm{z}}$ bipolar crossing exhibited two spiral nulls connected by a separator as well. The two nulls were both outside of the spacecraft tetrahedron, and thus, the Poincare index corresponded to zero in this time interval (not shown). Previously, the separator model with two radial nulls suggested the X-line topology. ${ }^{5,11-14}$ However, the two spiral nulls linked by the separator led to a different $\mathrm{X}$-line configuration. In the cases shown in Fig. 3, two fan surfaces of the spiral nulls in conjunction with the separator and spines form a half-closure cell in which magnetic field lines were folded into the shape of a flux rope [see the thick purple lines in Figs. 3(a) and 3(b)]. It appears likely that the same flux rope was recorded twice by Cluster due to the flapping of the current sheet and results in the repeating $B_{z}$ bipolar signatures in Fig. 2.

\section{Two spiral nulls linked by both the separator and the spine to form a flux rope}

At 09:48 UT on 1st October, 2001, as shown in Figs. 4(a)-4(c), the bipolar signature of $\mathrm{B}_{\mathrm{z}}$ (in the brown shadow region) in conjunction with the increase in electron density and energetic electron flux was detected in the magnetotail (at $[-16.4,7.9,0.7] \mathrm{R}_{\mathrm{E}}$ in GSM coordinates). ${ }^{47}$ The presence of a small $\mathrm{B}_{\mathrm{y}}$ guide-field revealed that the magnetic structure corresponded to a flux rope. During the interval, $3 \mathrm{D}$ electron distribution measurements were available with a resolution of 3 spins per minute on $\mathrm{C} 2$ and provided the details of electron dynamics inside the flux rope [Fig. 4(g)]. The dataset in Fig. 4(g) is reconstructed from the 3D distribution measurement over the spin where every time stamp displays one azimuthal bin with a total of 16 bins. For every azimuthal bin, the polar bins were replaced by the data re-binned into 12 pitch angle bins. The first six panels from top to bottom in Fig. 4(g) correspond to pitch angle distributions for electrons with energies of $13 \mathrm{keV}-20 \mathrm{keV}, 8 \mathrm{keV}-13 \mathrm{keV}, 3 \mathrm{keV}-8 \mathrm{keV}, 1 \mathrm{keV}$ $-3 \mathrm{keV}, 300 \mathrm{eV}-1 \mathrm{keV}$, and $100 \mathrm{eV}-300 \mathrm{eV}$, respectively. The last panel of Fig. 4(g) corresponds to the energy spectrogram of electrons. The data gaps were due to the limited angular coverage of the instrument. At 09:48:40.4 UT, $B_{z}$ began to change its sign, $B_{x}$ exhibited a maximum of $\sim 24 \mathrm{nT}$, while the magnetic field in the lobe exceeded $35 \mathrm{nT}$ as measured by $\mathrm{C} 1$ (not shown), thereby implying that the 


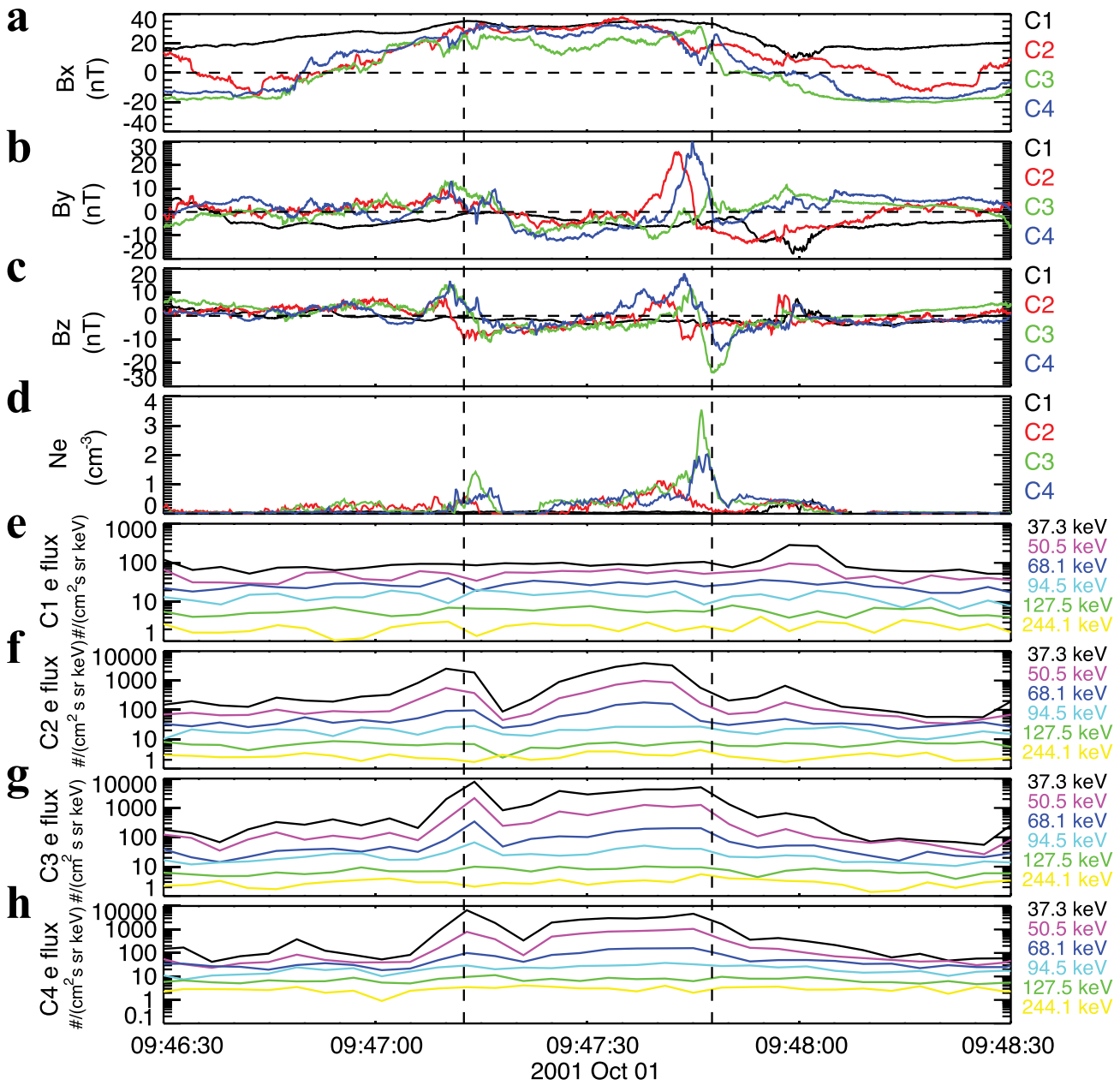

FIG. 2. Overview of the flux rope event in the magnetotail on 1st October, 2001. (a)-(c) Three components of the magnetic field for four Cluster spacecraft in the GSM coordinate. (d) Electron densities derived from spacecraft potential. (e)-(h) Differential energy flux of electrons with energies ranging from $37 \mathrm{keV}$ to $244 \mathrm{keV}$ for four Cluster spacecraft, respectively. Two vertical dashed lines represent the reconstruction times.

spacecraft was at the northern side of the flux rope center and at the leading edge of the flux rope. The first two panels of Fig. $4(\mathrm{~g})$ indicate that antiparallel electron beams with energy exceeding $8 \mathrm{keV}$ at the leading edge exist. It is not known whether a parallel electron beam exists since the parallel pitch angle was beyond the instrument's view. At around 09:48:43 UT, the magnetic strength $|\mathrm{B}|$ decreased to less than $10 \mathrm{nT}$, while $B_{z}$ had changed to a positive value. This indicated that $\mathrm{C} 2$ moved to the tailward edge of the flux rope and was considerably closer to the flux rope center. The flux of electrons detected by $\mathrm{C} 2$ with an energy higher than $3 \mathrm{keV}$ increased in all the available pitchangles (which range from $0^{\circ}$ to $120^{\circ}$, while the rest of the pitch angles were not covered by the sensor at that time). The measurement of the high-resolution electron energy spectrum suggested that the flux rope consisted of multiple layers with different electron properties.

The Poincaré index and the spiral index [Fig. 4(d)] also imply that spiral null points were present inside or near to the flux rope. The reconstruction results in Fig. 5 visualize the structure of the flux rope. The reconstruction time is shown in Fig. 4 by vertical dashed lines.
From the view angle displayed in Fig. 5(a), an $X$-line is shown on the tailward (right) side of the flux rope, and the perspective projection of the flux rope in the plot displays a loop-like configuration that can be often easily confused with an island. There was no magnetic null related to the $X$-line, and this was potentially because the nulls that formed the $X$-line were too far from the spacecraft to be reconstructed. The flux rope was embedded in the earthward outflow region and exhibited a size of approximately $1500 \mathrm{~km}\left(\mathrm{~d}_{\mathrm{i}} \sim 1140 \mathrm{~km}\right.$, while ion number density $\mathrm{n} \sim 0.04 \mathrm{~cm}^{-3}$ as obtained from CIS) in the outflow direction. The $X$-line was separated from the flux rope by approximately $2000 \mathrm{~km}$.

From another view angle of the reconstruction result, shown in Fig. 5(b), the $A_{s}$-spine- $B_{s}$ configuration was observed. Additionally, as shown in Fig. 5(c), the fan surfaces of the two spiral nulls are intersected at the separator to form a half-closure cell. The $A_{s}$-null was at $\sim[30,220,-730] \mathrm{km}$, and the $B_{s}$-null was at $\sim[-350,730,-730] \mathrm{km}$. The length of the "common spine" that connects the two spiral nulls is approximately $640 \mathrm{~km}$. The field lines near the spine line and inside 
$\mathbf{a}$

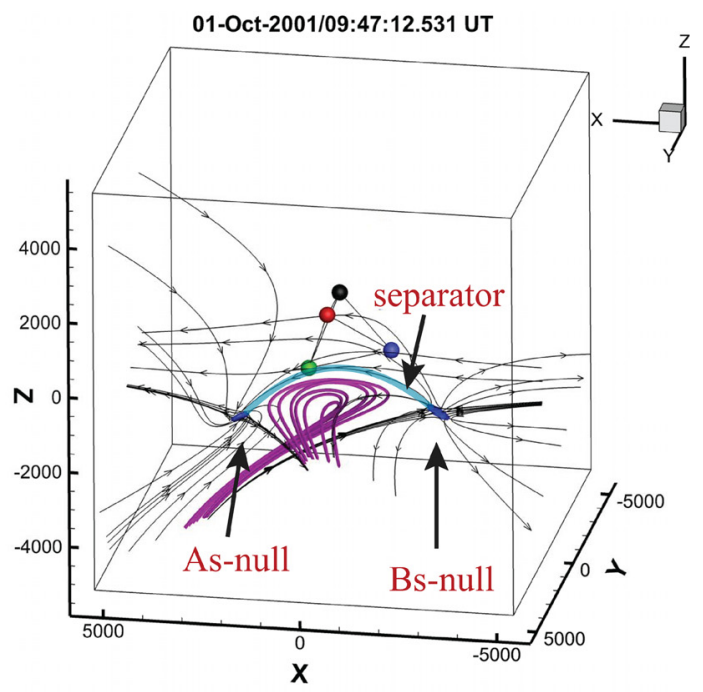

b

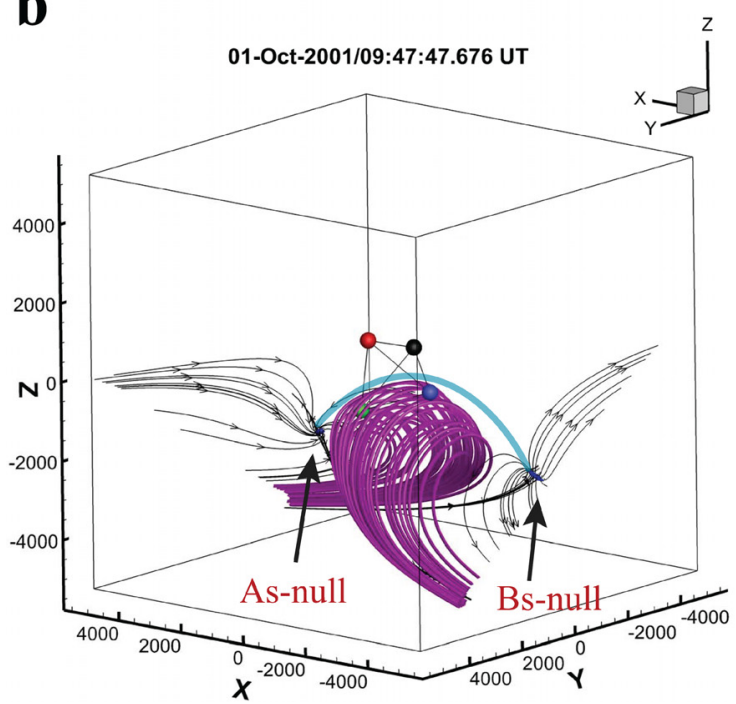

FIG. 3. Reconstruction results for the flux rope formed by spiral nulls linked by separator. The two reconstructions are obtained at the time marked by two vertical dashed lines in Fig. 2. The axes of coordinates are parallel to those of the GSM coordinate, while the original point is set at the center of the spacecraft tetrahedron. Colored spheres present the location of four Cluster satellites (black, red, green, and blue represent $\mathrm{C} 1, \mathrm{C} 2, \mathrm{C} 3$, and $\mathrm{C} 4$, respectively). Black curves correspond to constructed magnetic field lines. The arrows on the curves show the direction of the field lines. Both configurations consist of a $B_{s}$-null and an $A_{s}$-null. The two spiral nulls are interlinked by the intersection lines of the fan surfaces. The field lines of fan surfaces are not significantly displayed in (b) to avoid overcomplexity, while we have confirmed that the two fan surfaces can be intersected. The thick purple curves correspond to the field lines to show the flux rope.

the half-closure cell were wrapped to form the flux rope [see the thick purple line in Fig. 5(b)]. Specifically, C2 was near the fan surface of the $B_{s}$-type null [see Fig. 5(c)]. The $\mathbf{E} \times \mathbf{B}$ drift velocity at C2 [Fig. 4(e)] contains a large component of $-\mathrm{V}_{\mathrm{y}}$ in a manner similar to the events shown in Ref. 33, and this implied that the magnetized electrons are a

b

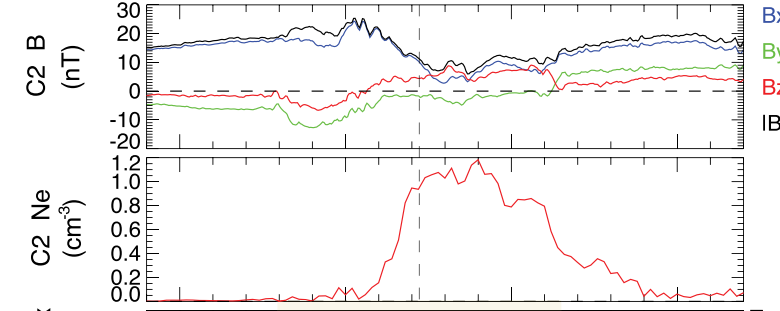

C

d

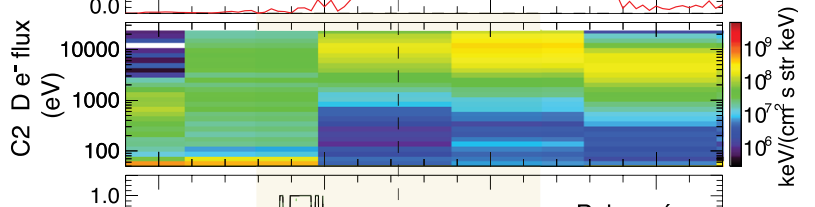

e

\section{$\mathrm{f}$}

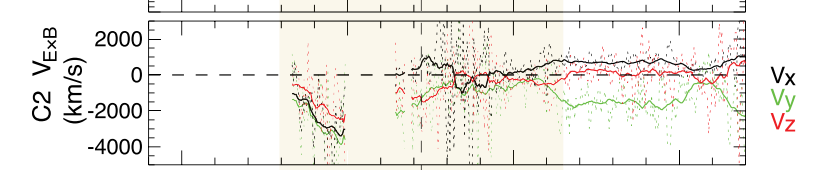

100

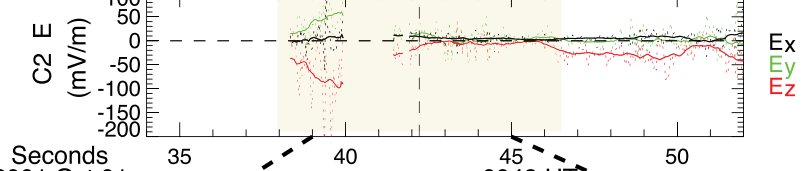

g
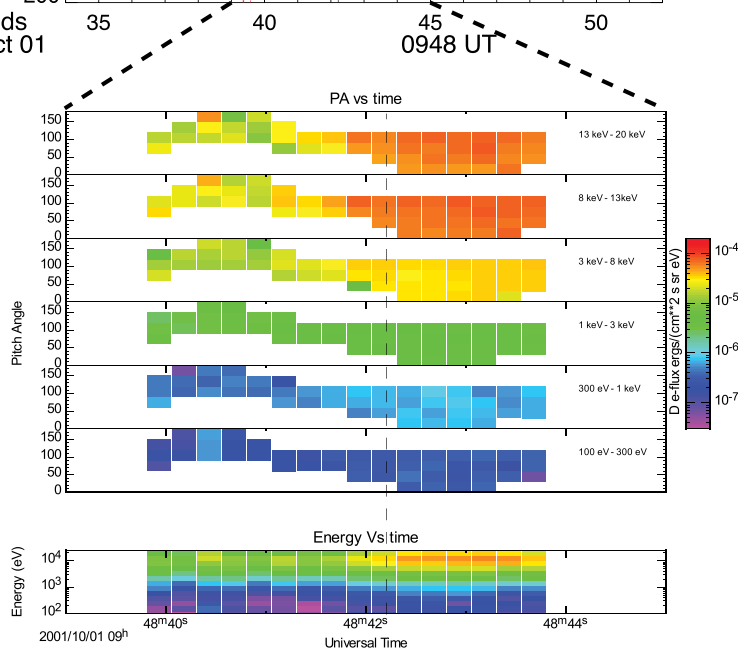

FIG. 4. Flux rope event observed by Cluster 2 at 09:48 UT on 1st October, 2001. (a) Magnetic field vector and strength for $\mathrm{C} 2$. (b) Electron density derived for spacecraft potential for $\mathrm{C} 2$. (c) Differential energy flux of electrons accumulated from all pitch-angles for $\mathrm{C} 2$. (d) The black line denotes the Poincaré index, and the dashed green line denotes the spiral index. (e) $\mathbf{E} \times \mathbf{B}$ drift velocity and (f) electric field for C2. The dotted lines in (f)-(i) denote the original data provided by CSA, and the solid lines denote the smoothed results of the original data. The smooth window corresponds to one second. The brown mask highlights the interval when $B_{z}$ exhibits a bipolar signal. The dashed vertical line denotes the times to perform reconstruction. The coordinate for all vectors is the GSM coordinate. (g) The dataset is reconstructed from the 3D distribution measurement over the spin where for every azimuthal bin, the polar zones are replaced by data re-binned into 12 pitch angle bins. The first six panels are the pitch angle distributions for electrons with energies of $13 \mathrm{keV}-20 \mathrm{keV}, 8 \mathrm{keV}-13 \mathrm{keV}, 3 \mathrm{keV}-8 \mathrm{keV}, 1 \mathrm{keV}-3 \mathrm{keV}, 300 \mathrm{eV}-1 \mathrm{keV}$, and $100 \mathrm{eV}-300 \mathrm{eV}$, from the top to bottom. The last panel is the energy spectrum of electrons. The white color denotes data gaps. 


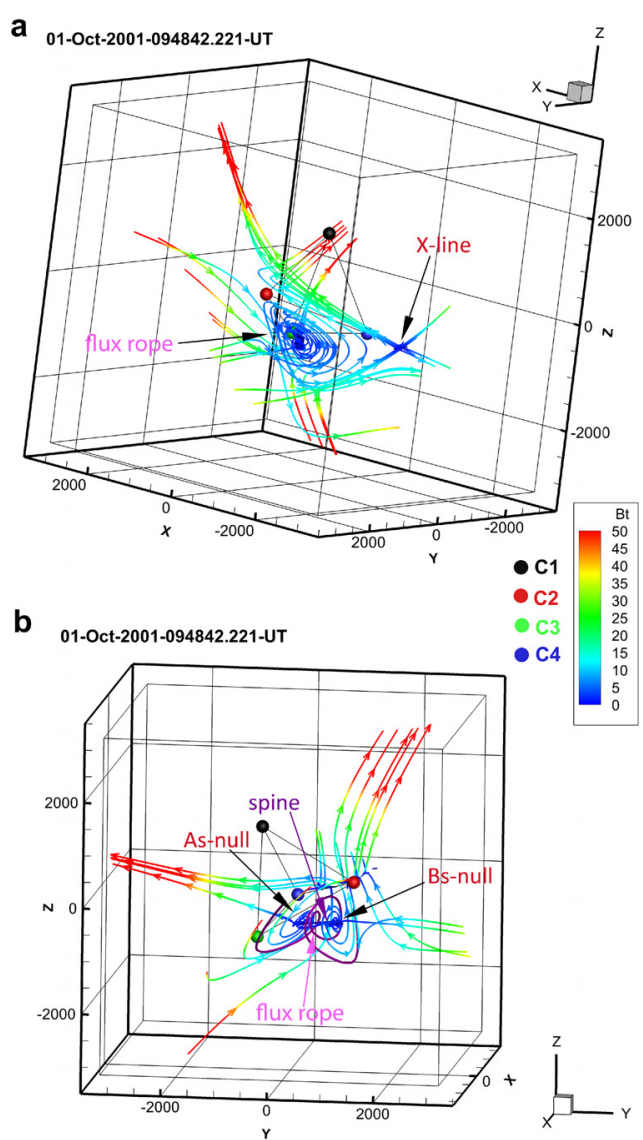

C

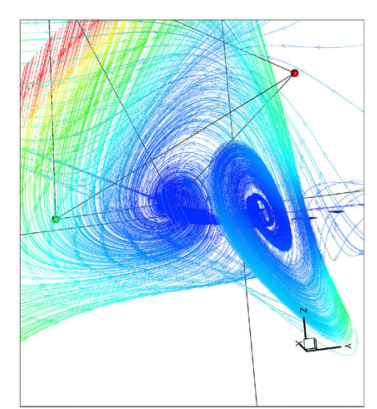

FIG. 5. Reconstruction result for the flux rope formed by spiral nulls linked by both the spine and the separator. (a) Magnetic configuration reconstructed at 09:48:42.221 UT. The time marked by the dashed line in Fig. 4. Colored spheres present the location of four Cluster satellites (black, red, green, and blue represent $\mathrm{C} 1, \mathrm{C} 2, \mathrm{C} 3$, and C4, respectively). The axes of coordinates are parallel to those of the GSM coordinate, while the original point is set at the center of the spacecraft tetrahedron. Colored curves correspond to constructed magnetic field lines. The arrows on the curves show the direction of the field lines. The flux rope displays a loop-like configuration. An X-line is located tailward of the flux rope with an approximate separation of $2000 \mathrm{~km}$. (b) Different view angles to display the same reconstruction result as that in (a). From this view angle, the configuration consists of a $B_{s}$-null and an $A_{s}$-null. The two spiral nulls are interlinked via their common spine that is approximately directed in the Y-direction. The thick purple curves also correspond to field lines to show the structure of the flux ropes. (c) Zoom-in view of the field lines near the two null points. flowing across the fan surface from the $B_{s}$-null to the $A_{s}$-null and are co-aligned with the spine line.

\section{THEORETICAL MODEL}

We observed three types of flux ropes through reconstruction of magnetic field configuration in the magnetotail based on the Cluster multispacecraft measurement. The flux ropes are all related to the spiral null pairs. The results indicate that two spiral nulls can be paired by the spine, or by the separator, or by both the spine and separator. All the structures can cause field lines to be wrapped up to exhibit flux rope features. In this section, we propose a theoretical model by modifying model (1) to catch the topology of the spiral null structures. The model is as follows:

$$
\left(B_{x}, B_{y}, B_{z}\right)=\left[x y-\frac{1}{2} j z+\varepsilon y, 1-y^{2}+\alpha x, z y+\frac{1}{2} j x\right] .
$$

In the model, the term $\alpha x$ represents the magnetic perturbation perpendicular to the null-null segment. When $\alpha=0$, the model is identical to model (1), the helically wrapped spine model. Evidently, when $\varepsilon$ is zero or small, the model exhibits the structure wherein two spiral null points are linked by their spine [see Fig. 6(a), with $\varepsilon=0, \alpha$ $=0$, and $j=6]$. However, if $\varepsilon$ becomes very large, the spine of two spiral nulls is separated notably and the two spiral nulls are not linked together (the configuration is similar to Fig. 3 in Ref. 38).

Furthermore, when $\varepsilon$ keeps small, but $\alpha$ is significant, the two spiral nulls can still be linked by the spine, while the two fan surfaces are also intersected to form a separator. This configuration is shown in Fig. 6(c) $(\varepsilon=0, \alpha=3$, and $j=6)$. The structure is similar to that analyzed in Ref. 38 in the third bifurcation example when the perturbation corresponds to a function of time and space. The structure shown in Fig. 5 is an example of this type.

Moreover, if both $\varepsilon$ and $\alpha$ are significant, the spines of two spiral nulls are separated and the fan surfaces are intersected at the separator as shown in Fig. 6(b) $(\varepsilon=2, \alpha=3$, and $j=6)$. The structures in Fig. 4 belong to this type. In the structure, the field lines are wrapped between the two fan surfaces [red lines in Fig. 6(b)]. The flux rope field lines in Figs. 6(a) and 6(c) are springlike and can form more than one loop. However, the red field lines in Fig. 6(b) can only exhibit one loop.

\section{DISCUSSIONS}

Previous studies mainly focus on the X-line formation in the light of the separator model, i.e., two radial nulls linked by the intersecting line of their fan surfaces. In this study, the results in Sec. II B indicate that the separator model also produces flux ropes with the two null points corresponding to spiral nulls. The key issue is that a strong current exists along the spine line, which makes the nulls correspond to spiral nulls and causes the field lines to be wrapped up. For example, given the model in Eq. (2), the Taylor expansion of magnetic field $\boldsymbol{B}$ at the center of the structure (i.e., at $[\mathrm{x}, \mathrm{y}, \mathrm{z}]=[0,0,0]$ ) gives $\boldsymbol{B}(\boldsymbol{r})=(0,1,0)+\boldsymbol{\delta} \boldsymbol{B} \cdot \boldsymbol{r}$. The first term of the Taylor expansion presents the magnetic guide field, which is aligned with the current density. The three eigenvalues of the matrix $\delta \boldsymbol{B}$ are 0 and $\pm \sqrt{ }\left(\varepsilon \alpha-j^{2} / 4\right)$. In a manner similar to Ref. 7 , the spiraling magnetic field requires that the two nonzero eigenvalues are complex, i.e., $\varepsilon \alpha-\mathrm{j}^{2} / 4<0$. When $\varepsilon \alpha$ is large [such as the case shown in Fig. 6(b)], the current density $j$ should exceed $2 \sqrt{ } \varepsilon \alpha$ to overcome the perturbation to form a 
a

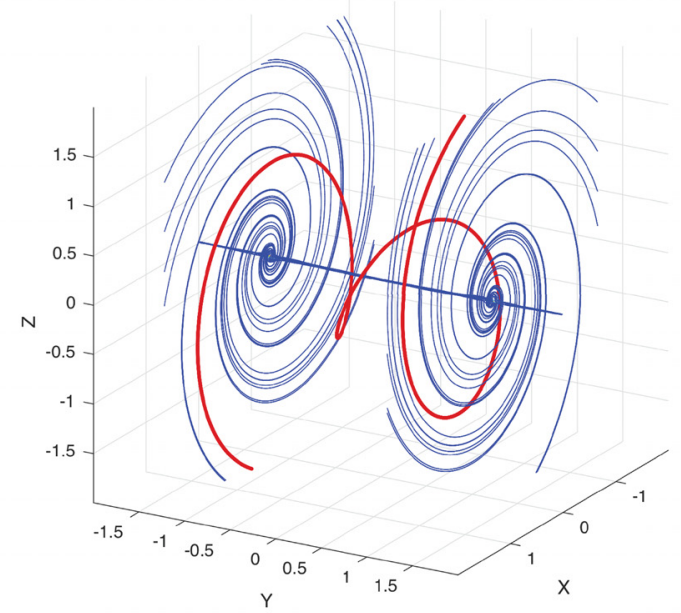

b
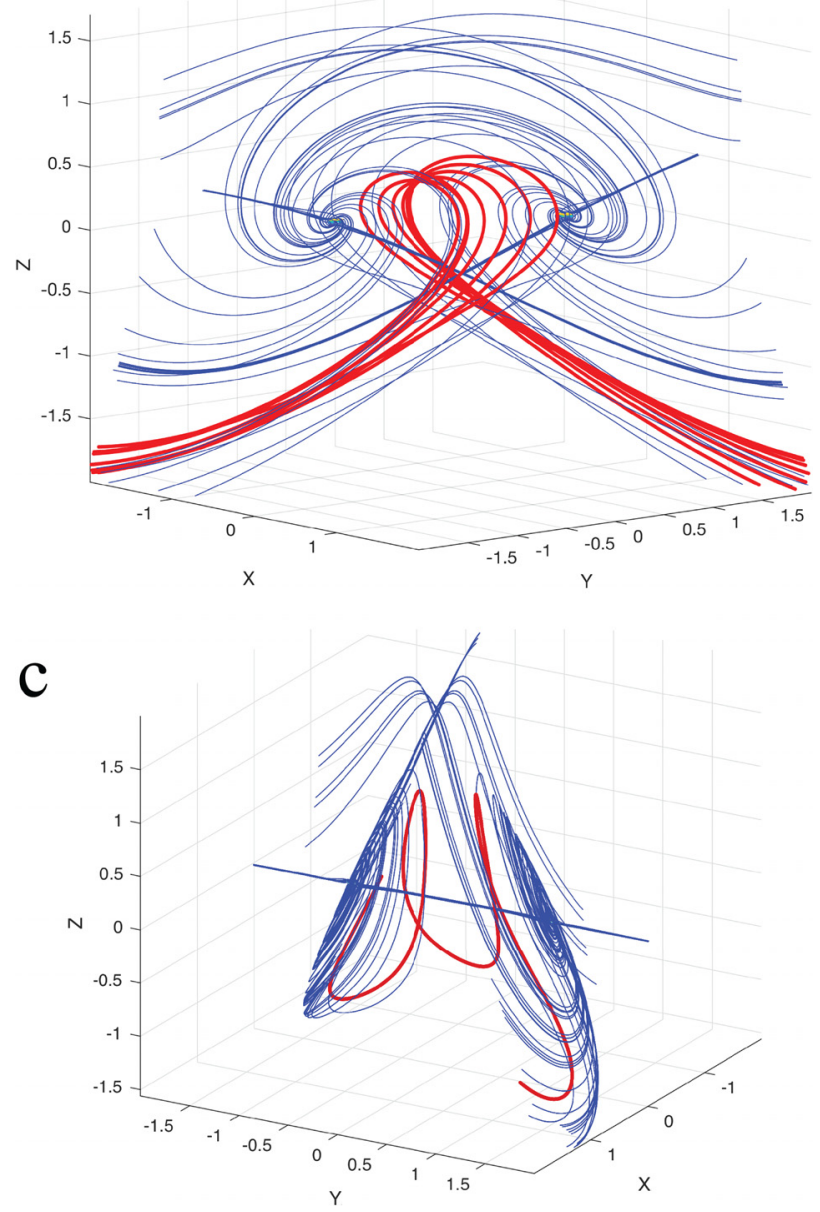

FIG. 6. Plots for three types of spiral null pairs based on the analytical model in Sec. III. Blue curves denote magnetic field lines adjacent to the null points. Red curves denote the magnetic field lines wrapped up to form flux ropes. Two spiral nulls are linked by the (a) spine line, (b) separator, and (c) both the spine and the separator. flux rope. In the first and the third type cases in this study, the spine lines connecting the two spiral nulls are helically wrapped together. Currents can flow directly from one null point to the other, and the field lines wrap around the spine lines to form the flux rope structure. More than two magnetic nulls can coexist in the reconnection region. ${ }^{34}$ The linkage pattern between any two of the multiple nulls can affect the magnetic topology and dynamics in the reconnection domain. For example, if the " $\mathrm{A}_{\mathrm{s}}$-spine- $\mathrm{B}_{\mathrm{s}}$-like" configuration in Sec. II A is part of a chain of spiral nulls in a manner similar to the simulation results shown in Ref. 32, then the flux ropes can extend far away from the spacecraft in the out-of-plane direction, and this makes the generation of flux rope significantly more complicated. ${ }^{3}$

It has been shown that flux ropes and spiral nulls can be formed locally in the outflow region, which is observed as a secondary reconnection site. ${ }^{33}$ The flux rope is also observed in the separatrix region. ${ }^{48}$ In $3 \mathrm{D}$ simulation results, ${ }^{27}$ flux ropes can be formed in arbitrary places in the reconnection region, while only one X-line can be clearly recognized. Additionally, the evolution of flux ropes can lead to a turbulent process where electron physics plays a central role. ${ }^{27}$ The flux rope shown in Sec. II C is located in the outflow region, and only one X-line is found in this region. The two spiral nulls are linked by both the spine and the separator and correspond to the case with large $\alpha$ in model (2). This configuration can potentially evolve from the helically wrapped spine model (negligible $\alpha$ ) if a large perturbation perpendicular to the spine exists. Reference 38 indicated that a degenerated null can bifurcate into different types of null pairs when the magnetic perturbation changes with time and space, including the first case and the third case described above in our model. The reconnection region is highly dynamic, and the magnetic perturbations can vary quickly. Thus, we speculate that, during the reconnection processes with disturbances inside the reconnection region, the different types of spiral null pairs can evolve between each other along with variations of the perturbations. The change in the linkage type can also change the magnetic topology and morphology, and this modifies the plasma properties inside the flux rope. The observation and simulation results suggest that the flux ropes can be formed without multiple X-lines, and the proposed model (2) implies that different flux ropes may be in different evolution stages related to the turbulent magnetic region.

Small-scale flux ropes and spiral nulls are commonly observed in the reconnection region in both space plasma and simulations. It is widely known that flux ropes, spiral nulls, and turbulence are closely related to each other, and they all play roles in the evolution of reconnection process. ${ }^{27,33,34}$ The present study shows that the null points and flux ropes are indispensable features of the reconnection region. The cases in Secs. II B and II C are observed within a short duration of two minutes and have been reported to be separated by multiple reconnection sites. ${ }^{47}$ The different linkage types of the null points for the two cases may expose different magnetic perturbation environments in different reconnection domains. Additionally, the spiral null pair and the flux rope could experience different types of perturbations during their transport away from the $\mathrm{X}$-line after their formation in the outflow region, during which the electrons can get accelerated. The potential transformation between the three types of linkages also implies the possibility that the flux ropes described in Secs. II and III were potentially at different evolution stages. 


\section{CONCLUSION}

The study explored three types of magnetic null pairs with different linkage modes to form flux ropes in the magnetotail reconnection region. The result implies that the null points play a vital role in the reconnection processes, not only in the $\mathrm{X}$-line configuration but also in the formation of flux ropes. In the latter case, the two null points are both spiral ones that are linked by a spine, or by a separator, or by a combination of a spine and a separator. All three types of spiral null pairs can be summed up into an analytical model as expressed by Eq. (2). The observational results and the model indicate that the two nulls and their fan surfaces serve as the boundaries of the flux rope. A distinct characteristic is the number of the loops. The separator configuration shown in Figs. 3 and 6(b) has just one loop. With respect to the other two configurations with helically wrapped spines, the loop number exceeds one and can be determined by the current density along the spine and the distance between the two nulls. Differences in the flux rope size and loop number can lead to different particle trajectories and acceleration processes. Furthermore, as shown in Eq. (2), different types of magnetic perturbations can lead to different types of linkages between nulls.

Different linkages between null points lead to the diversity of the flux ropes regarding the loop number and the boundary, indicating that the $3 \mathrm{D}$ reconnection is a highly dynamical process with complex geometry. The investigation of null points and their linkage is extremely helpful in the understanding of the nature of $3 \mathrm{D}$ reconnection. In order to understand the evolution of the flux ropes and magnetic null pairs, it is necessary to conduct statistical studies using in situ data from spacecraft or studies of the solar active regions using continuous images from the telescope(s) on the ground or in space.

\section{ACKNOWLEDGMENTS}

This work was supported by the Strategic Priority Research Program of Chinese Academy of Sciences (Grant No. XDA17010201). This study was supported by the National Natural Science Foundation of China (41704169, 41525016, and 41274167). Z. Y. acknowledges financial support from the Belgian Federal Science Policy Office (BELSPO) via the PRODEX Program of ESA. The authors acknowledge the Cluster PEACE, CIS, FGM, and EFW instrument teams for the provision of the data and the Cluster CSA for providing access to the data. The authors thank the International Space Science Institute in Bern, Switzerland, its staff, and directors for partial support through the ISSI Team (from Cluster to MMS).

\section{REFERENCES}

${ }^{1}$ P. A. Sweet, Il Nuovo Cimento (1955-1965) 8(2), 188-196 (1958).

${ }^{2}$ E. N. Parker, Astrophys. J., Suppl. Ser. 8, 177 (1963).

${ }^{3}$ J. M. Greene, J. Geophysical Res. 93(A8), 8583-8590 (1988).

${ }^{4} \mathrm{~K}$. Galsgaard and A. Nordlund, J. Geophysical Res.: Space Phys. 102(A1), 231-248 (1997).

${ }^{5}$ C. J. Xiao, X. G. Wang, Z. Y. Pu, Z. W. Ma, H. Zhao, G. P. Zhou, J. X. Wang, M. G. Kivelson, S. Y. Fu, Z. X. Liu, Q. G. Zong, M. W. Dunlop, K. H. Glassmeier, E. Lucek, H. Reme, I. Dandouras, and C. P. Escoubet, Nat. Phys. 3(9), 609-613 (2007).

${ }^{6}$ C. J. Xiao, X. G. Wang, Z. Y. Pu, H. Zhao, J. X. Wang, Z. W. Ma, S. Y. Fu, M. G. Kivelson, Z. X. Liu, Q. G. Zong, K. H. Glassmeier, A. Balogh, A. Korth, H. Reme, and C. P. Escoubet, Nat. Phys. 2(7), 478-483 (2006).
${ }^{7}$ Y.-T. Lau and J. M. Finn, Astrophys. J., Part 1 350, 672-691 (1990).

${ }^{8}$ J. M. Greene, J. Comput. Phys. 98(2), 194-198 (1992).

${ }^{9}$ E. R. Priest and V. S. Titov, "Philosophical Transactions of the Royal Society of London,” Ser. A: Math., Phys. Eng. Sci. 354(1721), 2951-2992 (1996).

${ }^{10}$ E. R. Priest and D. I. Pontin, Phys. Plasmas 16(12), 122101 (2009).

${ }^{11}$ J. S. He, Q. G. Zong, X. H. Deng, C. Y. Tu, C. J. Xiao, X. G. Wang, Z. W. Ma, Z. Y. Pu, E. Lucek, A. Pedersen, A. Fazakerley, N. Cornilleau-Wehrlin, M. W. Dunlop, H. Tian, S. Yao, B. Tan, S. Y. Fu, K. H. Glassmeier, H. Reme, I. Dandouras, and C. P. Escoubet, Geophys. Res. Lett. 35(14), L14104, https:// doi.org/10.1029/2008GL034085 (2008).

${ }^{12}$ X. H. Deng, M. Zhou, S. Y. Li, W. Baumjohann, M. Andre, N. Cornilleau, O. Santolík, D. I. Pontin, H. Reme, E. Lucek, A. N. Fazakerley, P. Decreau, P. Daly, R. Nakamura, R. X. Tang, Y. H. Hu, Y. Pang, J. Büchner, H. Zhao, A. Vaivads, J. S. Pickett, C. S. Ng, X. Lin, S. Fu, Z. G. Yuan, Z. W. Su, and J. F. Wang, J. Geophys. Res.: Space Phys. 114(A7), A07216, https://doi.org/10.1029/2008JA013197 (2009).

${ }^{13}$ M. W. Dunlop, Q. H. Zhang, C. J. Xiao, J. S. He, Z. Pu, R. C. Fear, C. Shen, and C. P. Escoubet, Phys Rev Lett 102(7), 075005 (2009).

${ }^{14}$ R. L. Guo, Z. Y. Pu, C. J. Xiao, X. G. Wang, S. Y. Fu, L. Xie, Q. G. Zong, J. S. He, Z. H. Yao, J. Zhong, and J. X. Li, J. Geophys. Res.-Space Phys. 118(10), 6116-6126, https://doi.org/10.1002/jgra.50569 (2013).

${ }^{15}$ J. C. Dorelli, A. Bhattacharjee, and J. Raeder, J. Geophys. Res.: Space Phys. 112(A2), A02202, https://doi.org/10.1029/2006ja011877 (2007).

${ }^{16}$ J. A. Slavin, R. P. Lepping, J. Gjerloev, D. H. Fairfield, M. Hesse, C. J. Owen, M. B. Moldwin, T. Nagai, A. Ieda, and T. Mukai, J. Geophys. Res. 108(A1), 1015, https://doi.org/10.1029/2002JA009557 (2003).

${ }^{17}$ M. N. Rosenbluth, R. Z. Sagdeev, J. B. Taylor, and G. M. Zaslavski, Nucl. Fusion 6(4), 297-300 (1966).

${ }^{18}$ R. F. Gandy, G. J. Hartwell, J. D. Hanson, S. F. Knowlton, and H. Lin, Phys. Fluids B: Plasma Phys. 5(12), 4384-4390 (1993).

${ }^{19}$ F. Ebrahimi and R. Raman, Phys. Rev. Lett. 114(20), 205003 (2015).

${ }^{20}$ V. Archontis, K. Galsgaard, F. Moreno-Insertis, and A. W. Hood, Astrophys. J. 645(2), L161-L164 (2006).

${ }^{21}$ V. Archontis, A. W. Hood, and C. Brady, Astron. Astrophys. 466(1), 367-376 (2007).

${ }^{22}$ L. J. Chen, N. Bessho, B. Lefebvre, H. Vaith, A. Fazakerley, A. Bhattacharjee, P. A. Puhl-Quinn, A. Runov, Y. Khotyaintsev, A. Vaivads, E. Georgescu, and R. Torbert, J. Geophys. Res.: Space Phys. 113(A12), A12213, https://doi.org/ 10.1029/2008ja013385 (2008).

${ }^{23}$ R. Wang, Q. Lu, A. Du, and S. Wang, Phys. Rev. Lett. 104(17), 175003 (2010).

${ }^{24}$ C. T. Russell and R. C. Elphic, Space Sci. Rev. 22(6), 681-715 (1978).

${ }^{25}$ J. P. Eastwood, T. D. Phan, P. A. Cassak, D. J. Gershman, C. Haggerty, K. Malakit, M. A. Shay, R. Mistry, M. Oieroset, C. T. Russell, J. A. Slavin, M. R. Argall, L. A. Avanov, J. L. Burch, L. J. Chen, J. C. Dorelli, R. E. Ergun, B. L. Giles, Y. Khotyaintsev, B. Lavraud, P. A. Lindqvist, T. E. Moore, R. Nakamura, W. Paterson, C. Pollock, R. J. Strangeway, R. B. Torbert, and S. Wang, Geophys. Res. Lett. 43(10), 4716-4724, https://doi.org/10.1002/2016GL068747 (2016).

${ }^{26}$ W. L. Teh, R. E. Denton, B. U. O. Sonnerup, and C. Pollock, Geophys. Res. Lett. 44(13), 6517-6524, https://doi.org/10.1002/2017GL074291 (2017).

${ }^{27}$ W. Daughton, V. Roytershteyn, H. Karimabadi, L. Yin, B. J. Albright, B. Bergen, and K. J. Bowers, Nat. Phys. 7(7), 539-542 (2011).

${ }^{28}$ G. Lapenta, S. Markidis, M. V. Goldman, and D. L. Newman, Nat. Phys. 11(8), 690-695 (2015).

${ }^{29}$ H. Zhao, J. X. Wang, J. Zhang, C. J. Xiao, and H. M. Wang, Chin. J Astron. Astrophys. 8(2), 133-145 (2008).

${ }^{30}$ P. F. Wyper and D. I. Pontin, Phys. Plasmas 21(10), 102102 (2014).

${ }^{31}$ P. F. Wyper and D. I. Pontin, Phys. Plasmas 21(8), 082114 (2014).

${ }^{32}$ V. Olshevsky, A. Divin, E. Eriksson, S. Markidis, and G. Lapenta, Astrophys. J. 807(2), 155 (2015).

${ }^{33}$ R. Guo, Z. Pu, L.-J. Chen, S. Fu, L. Xie, X. Wang, M. Dunlop, Y. V. Bogdanova, Z. Yao, C. Xiao, J. He, and A. N. Fazakerley, Phys. Plasmas 23(5), 052901 (2016).

${ }^{34}$ R. Guo, Z. Pu, S. Fu, L. Xie, M. Dunlop, Y. V. Bogdanova, J. He, X. Wang, and Z. Yao, Sci. Bull. 61(14), 1145-1150 (2016).

${ }^{35}$ C. P. Escoubet, M. Fehringer, and M. Goldstein, Ann. Geophys. 19(10/12), 1197-1200 (2001).

${ }^{36}$ H. S. Fu, A. Vaivads, Y. V. Khotyaintsev, V. Olshevsky, M. André, J. B. Cao, S. Y. Huang, A. Retinò, and G. Lapenta, J. Geophys. Res.: Space Phys. 120(5), 3758-3782, https://doi.org/10.1002/2015JA021082 (2015). 
${ }^{37}$ E. Eriksson, A. Vaivads, Y. V. Khotyaintsev, V. M. Khotyayintsev, and M. André, Geophys. Res. Lett. 42(17), 6883-6889, https://doi.org/10.1002/ 2015GL064959 (2015).

${ }^{38}$ N. A. Murphy, C. E. Parnell, and A. L. Haynes, Phys. Plasmas 22(10), 102117 (2015).

${ }^{39}$ J. S. He, C. Y. Tu, H. Tian, C. J. Xiao, X. G. Wang, Z. Y. Pu, Z. W. Ma, M. W. Dunlop, H. Zhao, G. P. Zhou, J. X. Wang, S. Y. Fu, Z. X. Liu, Q. G. Zong, K. H. Glassmeier, H. Reme, I. Dandouras, and C. P. Escoubet, J. Geophys. Res.: Space Phys. 113(A5), A05205, https://doi.org/10.1029/ 2007ja012609 (2008).

${ }^{40}$ A. Balogh, C. M. Carr, M. H. Acuña, M. W. Dunlop, T. J. Beek, P. Brown, K. H. Fornacon, E. Georgescu, K. H. Glassmeier, J. Harris, G. Musmann, T. Oddy, and K. Schwingenschuh, Ann. Geophys. 19(10/12), 1207-1217 (2001).

${ }^{41}$ H. Rème, C. Aoustin, J. M. Bosqued, I. Dandouras, B. Lavraud, J. A. Sauvaud, A. Barthe, J. Bouyssou, T. Camus, O. Coeur-Joly, A. Cros, J. Cuvilo, F. Ducay, Y. Garbarowitz, J. L. Medale, E. Penou, H. Perrier, D. Romefort, J. Rouzaud, C. Vallat, D. Alcaydé, C. Jacquey, C. Mazelle, C. d’Uston, E. Möbius, L. M. Kistler, K. Crocker, M. Granoff, C. Mouikis, M. Popecki, M. Vosbury, B. Klecker, D. Hovestadt, H. Kucharek, E. Kuenneth, G. Paschmann, M. Scholer, N. Sckopke, E. Seidenschwang, C. W. Carlson, D. W. Curtis, C. Ingraham, R. P. Lin, J. P. McFadden, G. K. Parks, T. Phan, V. Formisano, E. Amata, M. B. BavassanoCattaneo, P. Baldetti, R. Bruno, G. Chionchio, A. Di Lellis, M. F. Marcucci, G. Pallocchia, A. Korth, P. W. Daly, B. Graeve, H. Rosenbauer, V. Vasyliunas, M. McCarthy, M. Wilber, L. Eliasson, R. Lundin, S. Olsen, E. G. Shelley, S. Fuselier, A. G. Ghielmetti, W. Lennartsson, C. P. Escoubet, H. Balsiger, R. Friedel, J. B. Cao, R. A. Kovrazhkin, I. Papamastorakis, R. Pellat, J. Scudder, and B. Sonnerup, Ann. Geophys. 19(10/12), 1303-1354 (2001).
${ }^{42}$ A. D. Johnstone, C. Alsop, S. Burge, P. J. Carter, A. J. Coates, A. J. Coker, A. N. Fazakerley, M. Grande, R. A. Gowen, C. Gurgiolo, B. K. Hancock, B. Narheim, A. Preece, P. H. Sheather, J. D. Winningham, and R. D. Woodliffe, Space Sci. Rev. 79(1), 351-398 (1997).

${ }^{43}$ A. N. Fazakerley, A. D. Lahiff, R. J. Wilson, I. Rozum, C. Anekallu, M. West, and H. Bacai, in The Cluster Active Archive: Studying the Earth's Space Plasma Environment, edited by H. Laakso, M. Taylor, and C. P. Escoubet (Springer Netherlands, Dordrecht, 2010), pp. 129-144.

${ }^{44}$ B. Wilken, W. I. Axford, I. Daglis, P. Daly, W. GÜTtler, W. H. Ip, A. Korth, G. Kremser, S. Livi, V. M. Vasyliunas, J. Woch, D. Baker, R. D. Belian, J. B. Blake, J. F. Fennell, L. R. Lyons, H. Borg, T. A. Fritz, F. Gliem, R. Rathje, M. Grande, D. Hall, K. KecsuemÉTy, S. McKenna-Lawlor, K. Mursula, P. Tanskanen, Z. $\mathrm{Pu}$, I. Sandahl, E. T. Sarris, M. Scholer, M. Schulz, F. SØRass, and S. Ullaland, Space Sci. Rev. 79(1), 399-473 (1997).

${ }^{45}$ A. Pedersen, B. Lybekk, M. André, A. Eriksson, A. Masson, F. S. Mozer, P. A. Lindqvist, P. M. E. Décréau, I. Dandouras, J. A. Sauvaud, A. Fazakerley, M. Taylor, G. Paschmann, K. R. Svenes, K. Torkar, and E. Whipple, J. Geophys. Res. Space Phys. 113(A7), A07S33, https://doi.org/10.1029/2007ja012636 (2008).

${ }^{46}$ H. Zhao, J. X. Wang, J. Zhang, and C. J. Xiao, Chin. J. Astron. Astrophys. 5(5), 443-447 (2005).

${ }^{47}$ L. J. Chen, A. Bhattacharjee, P. A. Puhl-Quinn, H. Yang, N. Bessho, S. Imada, S. Mühlbachler, P. W. Daly, B. Lefebvre, Y. Khotyaintsev, A. Vaivads, A. Fazakerley, and E. Georgescu, Nat. Phys. 4(1), 19-23 (2007).

${ }^{48}$ S. Y. Huang, A. Retino, T. D. Phan, W. Daughton, A. Vaivads, H. Karimabadi, M. Zhou, F. Sahraoui, G. L. Li, Z. G. Yuan, X. H. Deng, H. S. Fu, S. Fu, Y. Pang, and D. D. Wang, J. Geophys. Res.: Space Phys. 121(1), 205-213, https:// doi.org/10.1002/2015JA021468 (2016). 